\title{
Estructura y diversidad de especies arbóreas en bosques templados de San Luis Potosí, México
}

\section{Structure and diversity of arboreal species in temperate forests of San Luis Potosi, Mexico}

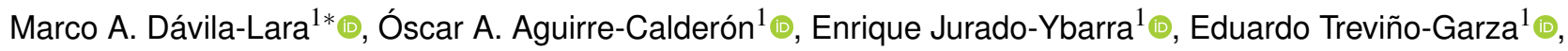 \\ Marco A. González-Tagle ${ }^{1}$ (D), Guillermo Trincado ${ }^{2}$ (D) \\ ${ }^{1}$ Facultad de Ciencias Forestales, Universidad Autónoma de Nuevo León, Carretera Nacional Km. 145, Apartado Postal 41, CP. 67700, \\ Linares, Nuevo León, México. \\ ${ }^{2}$ Universidad Austral de Chile. Instituto de Manejo Forestal, Casilla 567, Valdivia, Chile. \\ *Autor de correspondencia: marco.davila147@gmail.com
}

Artículo científico recibido: 31 de enero de 2019 aceptado: 17 de junio de 2019

RESUMEN. El estado de San Luis Potosí cuenta con ecosistemas boscosos que tienen limitada investigación en el ámbito forestal. Los estudios sobre la estructura y diversidad arbórea se realizan para generar información necesaria para efectuar un manejo conveniente en estos ecosistemas. El objetivo de la investigación fue evaluar y comparar la estructura y diversidad de especies forestales para dos condiciones de bosque templado bajo manejo forestal en San Luis Potosí. Se realizó un muestreo aleatorio para lo cual se establecieron tres parcelas de muestreo de $2500 \mathrm{~m}^{2}$ en cada localidad. Se obtuvo información dasométrica de individuos mayores de $7.5 \mathrm{~cm}$ de diámetro normal y se determinó la composición de especies, densidad por hectárea, abundancia, dominancia, frecuencia, índice de valor de importancia (IVI) e índice de distribución vertical (A). Para la UMA San Rafael se registraron 323 individuos, de cuatro familias, cuatro géneros y 10 especies; la abundancia fue de 430 árboles ha ${ }^{-1}$; siendo Pinus teocote la especie con mayor IVI (71.02\%). En el Predio San Joaquín se registraron 277 individuos, que pertenecen a tres familias, tres géneros y ocho especies; la abundancia fue de 369 árboles $\mathrm{ha}^{-1}$; P. devoniana fue la especie de mayor IVI (98.92\%). La riqueza específica fue de 10 y 8 especies, con índice de Margalef de 1.55 y 1.24. En relación con la diversidad del área, el índice de Shannon ( $\left.H^{\prime}\right)$ tuvo un valor de 1.91 y 1.51 , respectivamente. P. devoniana es la especie con mayor dominancia en ambas localidades con 4.04 y $6.62 \mathrm{~m}^{2} \mathrm{ha}^{-1}$.

Palabras clave: Bosques templados, composición, distribución vertical, índices de diversidad, manejo forestal.

ABSTRACT. The state of San Luis Potosí has forested ecosystems that have limited research in forestry. Studies on tree structure and diversity are carried out to generate information necessary to carry out a suitable management in these ecosystems. The objective of the research was to evaluate and compare the structure and diversity of forest species for two temperate forest conditions under forest management in San Luis Potosí. A random sampling was carried out for which three sampling plots of $2500 \mathrm{~m}^{2}$ were established in each locality. Densometric information was obtained from individuals larger than $7.5 \mathrm{~cm}$ in normal diameter and the species composition, density per hectare, abundance, dominance, frequency, Importance Value Index (IVI) and vertical distribution index (A) were determined. For UMA San Rafael 323 individuals were registered, of four families, four genera and 10 species; the abundance was 430 trees ha ${ }^{-1}$; being Pinus teocote the species with the highest IVI (71.02\%). In the San Joaquin Land, 277 individuals were registered, belonging to three families, three genera and eight species; the abundance was 369 trees ha ${ }^{-1}$; P. devoniana was the highest IVI species (98.92\%). The specific richness was of 10 and 8 species, with a Margalef index of 1.55 and 1.24. In relation to the diversity of the area, the Shannon index $\left(\mathrm{H}^{\prime}\right)$ had a value of 1.91 and 1.51 , respectively. P. devoniana is the species with greater dominance in both locations with 4.04 and $6.62 \mathrm{~m}^{2}$ ha $^{-1}$.

Key words: Temperate forests, composition, vertical distribution, diversity indexes, forest management. 


\section{INTRODUCCIÓN}

La región fisiográfica denominada Sierra Madre Oriental está constituida por un conjunto de serranías, las cuales se extienden dentro de los paralelos 20 a cerca del 30 , por lo que está en contacto con los sistemas atmosféricos más importantes de la región, lo que causa complejos mosaicos climáticos con altas precipitaciones. Por consecuencia, la vegetación presente en este sistema montañoso es altamente diversa, incluye matorrales xerófilos, bosque de coníferas y encinos, bosque mesófilo de montaña y selva tropical (Luna et al. 2004). La estructura de un ecosistema se define como la distribución de las principales características de la vegetación en el espacio, de especial importancia es la distribución de las especies por clases dimensionales (Li et al. 2014). Por otro lado, la estructura espacial de una masa es una característica que debe considerarse para una correcta planificación del manejo de los recursos forestales (Wehenkel et al. 2014). La caracterización estructural es importante para entender el funcionamiento de los ecosistemas, lo cual puede contribuir a los elementos incluidos dentro del manejo adecuado de los bosques (Castellanos-Bolaños et al. 2008).

El conocimiento de la estructura vertical y horizontal es esencial para el desarrollo de buenas prácticas de manejo forestal para la conservación de la biodiversidad en ecosistemas templados (AguirreCalderón 2015). Por lo anterior, el análisis de la distribución espacial, composición y estructura de una comunidad vegetal se ha incrementado entre los científicos y gestores de los recursos naturales, por ser el punto de partida para la correcta planeación de los programas de manejo, conservación y restauración ecológica (Gadow et al. 2012, Ni et al. 2014).

En México se han desarrollado estudios que evalúan la estructura y diversidad de comunidades vegetales, siendo los ecosistemas de clima templado los más estudiados, al respecto González et al. (2007) reportan la reconstrucción del historial de incendios y la estructura forestal que se deriva, dentro de bosques mixtos de pino encino en la Sierra Madre Oriental. Mientras que González et al. (2008) traba- jaron sobre los tipos de especies distribuidas en el ecosistema a lo largo de una cronosecuencia post incendio en un bosque mixto de Pino-Encino, siendo Quercus rysophylla la especie dominante después del incendio; pero 18 años después dominaron las especies de pino, como Pinus pseudostrobus y $P$. teocote. En tanto que Mora-Donjuán et al. (2016), destacan que los grados de comparación espacial dentro de la estructura de vegetación para diferentes sitios de matorral, depende de la competencia que se puede dar, ya sea de manera intraespecífica o interespecífica, además de la dominancia espacial que tiene cada individuo. Mientras que Graciano-Ávila et al. (2017), en un estudio de composición, estructura y diversidad de especies arbóreas en un bosque templado del estado de Durango, registran como especie dominante a $P$. cooperi.

En San Luis Potosí los bosques de Pinus y Quercus, se localizan en la porción más alta de la Sierra de Camarón y el cordón de la Mesa Larga (INEGI 2002). Pero son pocos los estudios de estructura y diversidad de especies para bosques templados, al respecto Leija-Loredo et al. (2011), reportan la distribución y dinámica espacio - tiempo del bosque de niebla. Mientras que Fortanelli et al. (2014), describen una lista de cerca de 199 especies, pertenecientes a 165 géneros y 91 familias, en una estructura de tres estratos (arbóreo, arbustivo y herbáceo). Por lo anterior, el objetivo de esta investigación fue evaluar y comparar la estructura y diversidad de especies arbóreas para dos condiciones de bosque templado en San Luis Potosí.

\section{MATERIALES Y MÉTODOS}

\section{Localización del área de estudio}

La investigación se realizó en las estribaciones de la Sierra Madre Oriental, en la Sierra de Álvarez y Sierra de Camarón, del estado de San Luis Potosí. Las parcelas se establecieron de forma cuadrada en dos sitios dentro del macizo montañoso; uno en la Unidad de Manejo Ambiental para la Conservación de la Vida Silvestre (UMA) San Rafael, en el municipio de Rioverde, dentro de las coordenadas $21^{\circ} 41^{\prime} 58.47^{\prime \prime}$ y $21^{\circ} 39^{\prime} 5.25^{\prime \prime}$ LN, $100^{\circ} 5^{\prime} 32.37^{\prime \prime}$ y $100^{\circ} 3^{\prime} 46.17^{\prime \prime}$ LO con 
un total de 648 ha. El segundo sitio se estableció en la Sierra de Camarón, en los límites del municipio de Santa María del Río, en el predio San Joaquín, en las coordenadas $21^{\circ} 40^{\prime} 10.9^{\prime \prime}$ y $21^{\circ} 39^{\prime} 58.3^{\prime \prime} \mathrm{LN}$ y $100^{\circ} 14^{\prime} 58.3^{\prime \prime}$ y $100^{\circ} 14^{\prime} 56.1$ " LO con un total de 766 ha (Figura 1).

El Predio San Joaquín se ubica en Sierra de Camarón, sobre un lecho rocoso, por lo que es frecuente observar afloramientos rocosos en el área (Fortanelli 1991), con elevación de 1800 a 2400 m (INEGI 2002). La UMA San Rafael se ubica en Sierra de Álvarez, se caracteriza por tener rocas sedimentarias, con elevación de 1500 a $1900 \mathrm{~m}$. El suelo es tipo Leptosol lítico (LPq) 100\%, somero, limitado en profundidad por roca dura continua o por una capa continua cementada dentro de una profundidad de $10 \mathrm{~cm}$ (INEGI 2002). Estos conjuntos de estribaciones tienen clima templado semiárido BS1hw (García 2004). La temperatura media anual es mayor a $18{ }^{\circ} \mathrm{C}$ y la precipitación media anual es de 1200 a 1800 mm (INEGI 2002).

La UMA San Rafael tiene 648 ha, es de conservación de venado cola blanca (Odocoileus virginianus), codorniz (Cyrtonix montezumae), guajolote silvestre (Meleagris gallopavo) y conejo silvestre (Sylvilagus floridanus), con vegetación de tipo bosque de pino-encino y bosque de encino. Dentro de la UMA se localiza un área con cerca de 230 ha de aprovechamiento a pequeña escala, de forma selectiva pero sin ningún tipo de método silvícola, donde se encuentran especies como $P$. teocote y $P$. devoniana. El predio San Joaquín tiene vegetación del tipo bosque de encino y bosque de pinoencino, tiene 766 ha en total de las cuales 631 ha tienen permiso para extracción maderable, las cuales por su configuración geográfica están bajo el método silvícola $\mathrm{MMOBI}$, el cual tiene la característica de aprovechar por selección, las especies con mayor aporte al volumen de extracción para el predio.

Se implementó un método de muestreo aleatorio en cada localidad, en el que el estrato de estudio fueron rodales de bosques mixtos de pino-encino y donde se realiza aprovechamiento maderable, el muestreo cubrió un área de $7500 \mathrm{~m}^{2}$, resulto del establecimiento de tres parcelas de $50 \times 50 \mathrm{~m}$ (2 500 $\mathrm{m}^{2}$ ), las cuales cubren más del $1 \%$ de la extensión de las áreas de aprovechamiento en ambas localidades en donde se aplicó la metodología recomendada por Corral et al. (2009). Dentro de las cuales se realizó un levantamiento de datos de altura y diámetro normal para individuos mayores a $7.5 \mathrm{~cm}$ de diámetro, se tomó a partir de este diámetro ya que son áreas de aprovechamiento por selección y por primera vez se establecerá una regeneración por plantación en ambas zonas. Adicionalmente se obtuvieron datos de azimut y distancia del centro de la parcela a cada individuo.

Para determinar la estructura horizontal se obtuvo la abundancia, de acuerdo al número de árboles, la dominancia de acuerdo al área basal y la frecuencia en base a la presencia dentro de cada sitio de muestreo, los cuales son parámetros que les dan el valor a las especies en la población (Mueller-Dombois et al. 1974). Los valores de estos parámetros, se usaron para calcular el índice de valor de importancia (IVI). Para la estructura vertical se toma en cuenta el índice de distribución vertical de especies (índice $A$ de Pretzch), el cual utiliza diferentes zonas de altura para la detección de cambios en la diversidad arbórea en los diferentes estratos del bosque, con el objetivo de aportar información básica sobre la dinámica del rodal (Pretzch 2009) (Tabla 1).

La abundancia, se calculó de acuerdo con el número de árboles mediante la fórmula:

$$
A_{i}=\frac{N_{i}}{E}, A R_{i}\left(\frac{A_{i}}{\sum_{i=1 \ldots n} A_{i}}\right) x 100
$$

Donde: $\mathrm{A}_{i}$ es la abundancia absoluta, $\mathrm{AR}_{i}$ es la abundancia relativa de la especie i respecto a la abundancia total, $\mathrm{N}_{i}$ es el número de individuos de la especie i, y $\mathrm{E}$ la superficie de muestreo (ha).

La dominancia absoluta y relativa se calculó en función del área basal, con la fórmula:

$$
D_{i}=\frac{A b_{i}}{E(h a)} \quad D R_{i}=\left(\frac{D_{i}}{\sum_{i=1 \ldots n} D_{1}}\right) \times 100
$$

Donde: $\mathrm{D}_{i}$ es la dominancia absoluta, $\mathrm{DR}_{i}$ es dominancia relativa de la especie i respecto a la dominancia, Ab el área basal de la especie i y E la superficie (ha). 


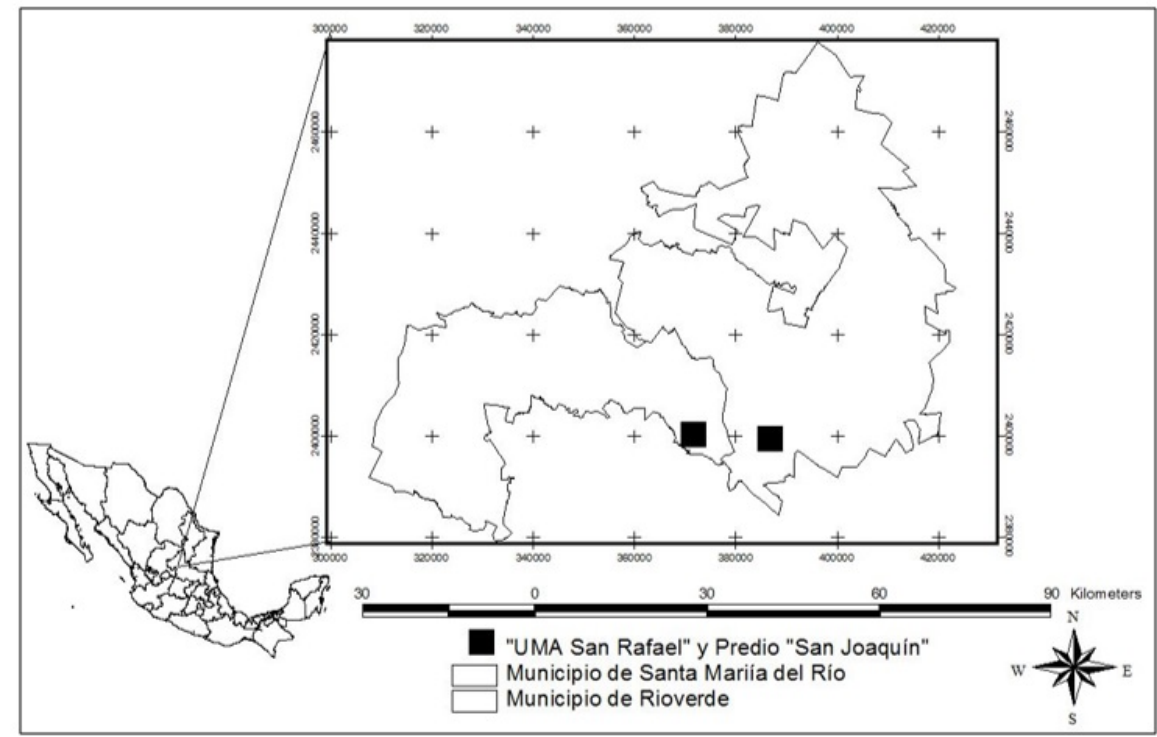

Figura 1. Localización de la UMA San Rafael y el predio San Joaquín.

Tabla 1. Parámetros y criterios utilizados para evaluar estructura vertical y horizontal dentro de las áreas de estudio.

\begin{tabular}{ccc}
\hline Estructura & Parámetro & Criterios \\
\hline Vertical & Índice de distribución vertical de especies & Diferentes estratos de altura. \\
& Abundancia & Número de árboles por ha \\
Horizontal & Dominancia & De acuerdo al área basal \\
& Frecuencia & En base a la presencia en cada sitio por especie \\
\hline
\end{tabular}

La frecuencia absoluta y relativa, se obtuvieron con la fórmula:

$$
F_{i}=\frac{P_{i}}{N S} \quad F R_{i}=\left(\frac{F_{i}}{\sum_{i=1 \ldots n} F_{1}}\right) x 100
$$

Donde: $\mathrm{F}_{i}$ es la frecuencia absoluta, $\mathrm{FR}_{i}$ es la frecuencia relativa de la especie i respecto a la suma de las frecuencias, $\mathrm{P}_{i}$ es el número de sitios en los que está presente la especie i y NS el número total de sitios de muestreo.

El índice de valor de importancia (IVI) se define como (Whittaker 1972, Moreno 2001):

$$
I V I=A R_{i}+D R_{i}+F R_{i}
$$

Para estimar la riqueza de especies se utilizó el índice de Margalef $\left(D_{m g}\right)$ (Margalef 1958) y para la diversidad alfa el índice de Shannon-Weaver $\left(\mathrm{H}^{\prime}\right)$ (Shannon 1948, Magurran 2004) mediante las fórmulas:

$$
\begin{gathered}
D_{m g}=\frac{(S-1)}{\operatorname{Ln}(N)} \\
H^{\prime}=-\sum_{i=i}^{S} p_{i} x \ln \left(p_{i}\right) \quad p i=\frac{n_{i}}{N}
\end{gathered}
$$

Donde: $\mathrm{S}$ es el número de especies presentes, $\mathrm{N}$ es el número total de individuos, $\mathrm{n}_{i}$ es el número de individuos de la especie i y $\mathrm{p}_{i}$ es la proporción de individuos de la especie i respecto al total de individuos.

El índice de Shannon toma en cuenta los componentes de la diversidad: número de especies y equidad, reflejando de mejor manera la diversidad florística de las poblaciones arbóreas.

Para la caracterización de la estructura vertical de las especies se utilizó el índice de distribución vertical de especies (Índice A de Pretzsch), el índice $A$ es una modificación del índice de Shannon en que tres estratos son representados de acuerdo a la altura máxima registrada (Aguirre 2002, Pretzsch 2009). 
El índice se estimó con la siguiente fórmula:

$$
A=-\sum_{i=1}^{S} \sum_{j=1}^{Z} P_{i j} x \ln \left(P_{i j}\right)
$$

Para la estimación, se definieron las zonas de altura (Jiménez et al. 2001): Estrato I: 80 a 100\% de la altura máxima del área, estrato II: 50 a $80 \%$ de la altura máxima, y estrato III: de cero a $50 \%$ de la altura máxima. Del índice $A$ se derivan el $A_{\max }$ que tiene valores entre cero y un valor máximo dado por el número de especies y zonas de altura; y $\mathrm{A}_{\text {rel }}$ que es la estandarización en porciento del índice $A$ (Rubio-Camacho et al. 2014).

$$
A_{\max }=\ln (S \times Z)
$$

Donde: $\mathbf{S}=$ número de especies presentes, $\mathbf{Z}=$ número de estratos de altura, $\mathrm{p}_{i j}=$ porcentaje de especies en cada zona que se estima mediante la siguiente ecuación $\mathrm{p}_{i j}=\mathrm{n}_{i ; j} / \mathrm{N}$. Siendo $\mathrm{n}_{i ; j}=$ número de individuos de la misma especie (i) en la zona (j) y $\mathrm{N}=$ número total de individuos. El valor de $\mathrm{A}$ se estandariza de la siguiente forma:

$$
A_{r e l}=\frac{A}{\ln (S \times Z)} \times 100
$$

\section{RESULTADOS}

Para la UMA San Rafael se registraron 323 individuos, de cuatro familias, cuatro géneros y 10 especies, siendo éstas Alnus acuminata, Arbutus xalapensis, P. devoniana, P. teocote, $Q$. affinis, $Q$. castanea, $Q$. crassifolia, $Q$. laeta, $Q$. resinosa y $Q$. viminea; para el Predio San Joaquín se registraron 277 individuos, que pertenecen a tres familias, tres géneros y ocho especies las cuales son $A$. xalapensis, P. devoniana, P. teocote, P. pseudostrobus, $Q$. crassifolia, $Q$. obtusata y $Q$. resinosa. La familia con mayor presencia en ambos sitios fue Fagaceae, con un género y seis especies para la UMA San Rafael y cuatro especies para el Predio San Joaquín. Esta constituye el $60 \%$ de la vegetación registrada en la UMA San Rafael y el $50 \%$ en San Joaquín.

La abundancia por género en la UMA San Rafael fue de 431 árboles ha $^{-1}$ y para el Predio San
Joaquín de 369 árboles ha ${ }^{-1}$, el género Quercus fue el más abundante dentro de la UMA San Rafael con 190 árboles ha ${ }^{-1}$ (44.27\%), mientras que el género Pinus destaco en el Predio San Joaquín con 280 árboles ha ${ }^{-1}(75.81 \%)$. La dominancia por género en área basal obtenida para la UMA San Rafael y el Predio San Joaquín fue de 18.86 y $18.98 \mathrm{~m}^{2} \mathrm{ha}^{-1}$, respectivamente. El género con mayor dominancia relativa para ambos sitios (9.23 y $15.74 \mathrm{~m}^{2} \mathrm{ha}^{-1}$ ) fue Pinus con 48.89 y $82.95 \%$. Los géneros con mayor frecuencia para la UMA San Rafael fueron Quercus y Pinus los cuales se registraron en la totalidad de las parcelas de muestreo (100\%). Para el predio San Joaquín la frecuencia fue uniforme, ya que los géneros Quercus, Pinus y Arbutus, tuvieron al menos un individuo presente dentro de las parcelas de muestreo (Tabla 2).

Las especies con mayor abundancia en la UMA San Rafael fueron $P$. teocote, $Q$. resinosa y $A$. xalapensis con 101, 88 y 72 árboles ha ${ }^{-1}$ (23.53, 20.43 y $16.72 \%$, respectivamente), las cuales comprenden el $60.68 \%$ de la vegetación presente en el área. Para el Predio San Joaquín las especies con mayor abundancia fueron $P$. devoniana, $P$. teocote y A. xalapensis con 163, 83 y 56 árboles ha $^{-1}$ (44.04, 22.38 y $15.16 \%$, respectivamente), la abundancia de estas especies equivalen al $81.58 \%$ del total de las especies del predio. La especie con mayor dominancia en área basal para la UMA San Rafael fue $P$. teocote $\left(5.19 \mathrm{~m}^{2} \mathrm{ha}^{-1}\right)$ con $27.49 \%$ y para el Predio San Joaquín fue P. devoniana $\left(6.62 \mathrm{~m}^{2} \mathrm{ha}^{-1}\right)$ con $34.88 \%$. La especie más frecuente en la UMA San Rafael fue $P$. teocote ( $100 \%$ de frecuencia absoluta) con $20 \%$ de frecuencia relativa y para el Predio San Joaquín fueron $P$. teocote y $P$. devoniana $(100 \%$ de frecuencia absoluta) con $20 \%$ de frecuencia relativa cada una. Una de las especie de menor frecuencia en ambos sitios fue $Q$. affinis que se encuentran en una sola parcela de cada sitio (33.33\% de abundancia absoluta cada una), con $6.67 \%$ de frecuencia relativa (Tabla 3).

El índice de valor de importancia en la UMA San Rafael indica que $P$. teocote $(71.02 \%)$ es la especie con mayor valor ecológico, para el Predio San Joaquín fue $P$. devoniana (98.92\%) la especie de 
Dávila-Lara et al.

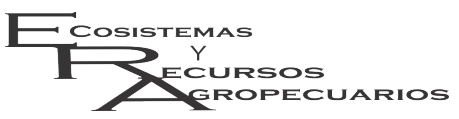

Diversidad en bosques de San Luis

Ecosist. Recur. Agropec.

6(18):399-409,2019

Tabla 2. Abundancia, dominancia, frecuencia e IVI por género registrados en los sitios de estudio.

\begin{tabular}{|c|c|c|c|c|c|c|c|}
\hline \multicolumn{8}{|c|}{ UMA San Rafael } \\
\hline \multirow[b]{2}{*}{ Género } & \multicolumn{2}{|c|}{ Abundancia } & \multicolumn{2}{|c|}{ Dominancia } & \multicolumn{2}{|c|}{ Frecuencia } & \multirow[b]{2}{*}{ IVI } \\
\hline & $\begin{array}{c}\text { Absoluta } \\
\mathrm{n} \mathrm{ha}^{-1}\end{array}$ & $\begin{array}{c}\text { Relativa } \\
(\%)\end{array}$ & $\begin{array}{l}\text { Absoluta } \\
\mathrm{m}^{2} \mathrm{ha}^{-1}\end{array}$ & $\begin{array}{c}\text { Relativa } \\
(\%)\end{array}$ & $\begin{array}{c}\text { Absoluta } \\
-\end{array}$ & $\begin{array}{c}\text { Relativa } \\
(\%)\end{array}$ & \\
\hline Quercus & 190.67 & 44.27 & 9.22 & 48.85 & 100.00 & 33.33 & 126.46 \\
\hline Pinus & 165.33 & 38.39 & 9.22 & 48.89 & 100.00 & 33.33 & 120.61 \\
\hline Arbutus & 72.00 & 16.72 & 0.41 & 2.18 & 66.67 & 22.22 & 41.12 \\
\hline \multirow[t]{2}{*}{ Alnus } & 2.67 & 60.62 & 0.01 & 0.08 & 33.33 & 11.11 & 11.81 \\
\hline & 430.67 & 100.00 & 18.86 & 100.00 & 300.00 & 100.00 & 300.00 \\
\hline \multicolumn{8}{|c|}{ Predio San Joaquín } \\
\hline \multirow[b]{2}{*}{ Género } & \multicolumn{2}{|c|}{ Abundancia } & \multicolumn{2}{|c|}{ Dominancia } & \multicolumn{2}{|c|}{ Frecuencia } & \multirow[b]{2}{*}{$\mathrm{IVI}$} \\
\hline & $\begin{array}{c}\text { Absoluta } \\
\mathrm{n} \mathrm{ha}^{-1}\end{array}$ & $\begin{array}{c}\text { Relativa } \\
(\%)\end{array}$ & $\begin{array}{l}\text { Absoluta } \\
\mathrm{m}^{2} \text { ha }^{-1}\end{array}$ & $\begin{array}{c}\text { Relativa } \\
\text { (\%) }\end{array}$ & $\begin{array}{c}\text { Absoluta } \\
-\end{array}$ & $\begin{array}{c}\text { Relativa } \\
\text { (\%) }\end{array}$ & \\
\hline Quercus & 33.33 & 9.03 & 3.11 & 16.37 & 100.00 & 33.33 & 58.73 \\
\hline Pinus & 280.00 & 75.81 & 15.74 & 82.95 & 100.00 & 33.33 & 192.09 \\
\hline \multirow[t]{2}{*}{ Arbutus } & 56.00 & 15.16 & 0.13 & 0.68 & 100.00 & 33.33 & 49.17 \\
\hline & 369.33 & 100.00 & 18.98 & 100.00 & 300.00 & 100.00 & 300.00 \\
\hline
\end{tabular}

Tabla 3. Abundancia, dominancia, frecuencia e IVI por especies registradas para las áreas de estudio.

\begin{tabular}{|c|c|c|c|c|c|c|c|}
\hline \multirow[b]{3}{*}{ Especie } & \multicolumn{6}{|c|}{ UMA San Rafael, Sierra de Álvarez, Rioverde } & \multirow[b]{3}{*}{ IVI } \\
\hline & \multicolumn{2}{|c|}{ Abundancia } & \multicolumn{2}{|c|}{ Dominancia } & \multicolumn{2}{|c|}{ Frecuencia } & \\
\hline & $\begin{array}{c}\text { Absoluta } \\
\mathrm{n} \mathrm{ha}\end{array}$ & $\begin{array}{c}\text { Relativa } \\
(\%)\end{array}$ & $\begin{array}{l}\text { Absoluta } \\
\mathrm{m}^{2} \mathrm{ha}^{-1}\end{array}$ & $\begin{array}{c}\text { Relativa } \\
(\%)\end{array}$ & $\begin{array}{c}\text { Absoluta } \\
-\end{array}$ & $\begin{array}{c}\text { Relativa } \\
\text { (\%) }\end{array}$ & \\
\hline P. teocote & 101.33 & 23.53 & 5.19 & 27.49 & 100.00 & 20.00 & 71.02 \\
\hline Q. resinosa & 88.00 & 20.43 & 3.79 & 20.08 & 66.67 & 13.33 & 53.85 \\
\hline A. xalapensis & 72.00 & 16.72 & 0.41 & 2.18 & 66.67 & 13.33 & 32.23 \\
\hline P. devoniana & 64.00 & 14.86 & 4.04 & 21.40 & 33.33 & 6.67 & 42.93 \\
\hline Q. crassifolia & 49.33 & 11.46 & 2.67 & 14.18 & 66.67 & 13.33 & 38.97 \\
\hline Q. castanea & 30.67 & 7.12 & 1.24 & 6.57 & 33.33 & 6.67 & 20.36 \\
\hline Q. affinis & 17.33 & 4.02 & 0.68 & 3.60 & 33.33 & 6.67 & 14.29 \\
\hline Q. laeta & 4.00 & 0.93 & 0.73 & 3.90 & 33.33 & 6.67 & 11.49 \\
\hline A. acuminata & 2.67 & 0.62 & 0.01 & 0.08 & 33.33 & 6.67 & 7.36 \\
\hline \multirow[t]{2}{*}{ Q. viminea } & 1.33 & 0.31 & 0.10 & 0.53 & 33.33 & 6.67 & 7.51 \\
\hline & 430.67 & 100.00 & 18.86 & 100.00 & 500.00 & 100.00 & 300.00 \\
\hline \multicolumn{8}{|c|}{ San Joaquín, Sierra de Camarón, Santa María del Río } \\
\hline & \multicolumn{2}{|c|}{ Abundancia } & \multicolumn{2}{|c|}{ Dominancia } & \multicolumn{2}{|c|}{ Frecuencia } & \\
\hline Especie & $\begin{array}{c}\text { Absoluta } \\
\mathrm{n} \mathrm{ha}^{-1}\end{array}$ & $\begin{array}{c}\text { Relativa } \\
(\%)\end{array}$ & $\begin{array}{l}\text { Absoluta } \\
\mathrm{m}^{2} \mathrm{ha}^{-1}\end{array}$ & $\begin{array}{c}\text { Relativa } \\
(\%)\end{array}$ & $\begin{array}{c}\text { Absoluta } \\
-\end{array}$ & $\begin{array}{c}\text { Relativa } \\
(\%)\end{array}$ & IVI \\
\hline P. devoniana & 162.67 & 44.04 & 6.62 & 34.88 & 100.00 & 20.00 & 98.92 \\
\hline P. teocote & 82.67 & 22.38 & 6.38 & 33.63 & 100.00 & 20.00 & 76.01 \\
\hline A. xalapensis & 56.00 & 15.16 & 0.13 & 0.68 & 66.67 & 13.33 & 29.17 \\
\hline P. pseudostrobus & 34.67 & 9.39 & 2.74 & 14.44 & 33.33 & 6.67 & 30.50 \\
\hline Q. crassifolia & 18.67 & 5.05 & 1.50 & 7.91 & 66.67 & 13.33 & 26.30 \\
\hline Q. obtusata & 12.00 & 3.25 & 0.78 & 4.13 & 66.67 & 13.33 & 20.71 \\
\hline Q. viminea & 1.33 & 0.36 & 0.60 & 3.19 & 33.33 & 6.67 & 10.22 \\
\hline \multirow[t]{2}{*}{ Q. resinosa } & 1.33 & 0.36 & 0.22 & 1.14 & 33.33 & 6.67 & 8.17 \\
\hline & 369.33 & 100.00 & 18.98 & 100.00 & 500.00 & 100.00 & 300.00 \\
\hline
\end{tabular}

mayor valor. La riqueza específica fue de 10 y 8 especies, con índice de Margalef de 1.55 y 1.24 . En relación con la diversidad del área, el índice de Shannon (H') tuvo un valor de 1.91 y 1.51 , respectivamente.

El índice de distribución vertical de las especies para la UMA San Rafael (Tabla 4) se definió en tres estratos: Estrato I o superior (30-24 m), el estrato II o intermedio $(24-15 \mathrm{~m})$ y el estrato III o inferior (15 - 0m); para el Predio San Joaquín los estratos fueron: Estrato I o superior (29 - $23.2 \mathrm{~m})$, el estrato II o intermedio (23.2 - $14.5 \mathrm{~m})$ y el estrato III o inferior (14.5 - 0m). Dentro de la UMA San Rafael el estrato superior está compuesto por $P$. devoniana con 8 árboles ha ${ }^{-1}$, lo que equivale al $1.86 \%$ de la zona, el estrato intermedio está compuesto por $P$. 
devoniana, P. teocote, $Q$. laeta, $Q$. crassifolia, y $Q$. viminea con 50 árboles ha ${ }^{-1}$, lo cual corresponde al $11.76 \%$ de la zona, y el estrato inferior se conformó por $Q$. resinosa, P. teocote, A. xalapensis, $Q$. crassifolia, P. devoniana, Q. castanea, $Q$. affinis y A. acuminata con 372 árboles ha ${ }^{-1}$, que equivale al $86.38 \%$ de la zona muestreada. Respecto al índice $A$, tiene un valor de $A=2.19$, una $A_{\max }=3.4$ y una $A_{r e l}=64.3 \%$, lo que indica que dentro de la UMA San Rafael, las especies se distribuyen mayormente dentro del estrato inferior y con gran número de individuos; el estrato inferior tiene una altura promedio de $7.65 \mathrm{~m}$, la que es cercano a la altura promedio de la muestra, que es $9.35 \mathrm{~m}$ e indica que el bosque es joven.

Tabla 4. Valores del índice vertical de Pretzsch para la UMA San Rafael.

\begin{tabular}{ccccc}
\hline & & \multicolumn{3}{c}{ Proporción (\%) } \\
\hline $100 \%-80 \%$ & $\mathrm{~N}$ & $\mathrm{~N} \mathrm{ha}^{-1}$ & Del total & En la zona \\
$(30 \mathrm{~m}-24 \mathrm{~m})$ & & & & \\
\hline Pinus devoniana & 6 & 8.0 & 100 & 1.86 \\
Suma & 6 & 8.0 & 100 & 1.86 \\
\hline $80 \%-50 \%$ & & & & \\
$(24 \mathrm{~m}-15 \mathrm{~m})$ & & & & 5.6 \\
\hline Pinus devoniana & 18 & 24.0 & 47.4 & 4.3 \\
Pinus teocote & 14 & 18.7 & 36.8 & 0.9 \\
Quercus laeta & 3 & 4.0 & 7.9 & 0.6 \\
Quercus crassifolia & 2 & 2.7 & 5.3 & 0.3 \\
Quercus viminea & 1 & 1.3 & 2.6 & 11.76 \\
Suma & 38 & 50.7 & 100.0 \\
\hline $50 \%$ - 0\% & & & & \\
$(15 \mathrm{~m}-$ Om $)$ & & & & 20.4 \\
\hline Quercus resinosa & 66 & 88.0 & 23.7 & 19.2 \\
Pinus teocote & 62 & 82.7 & 22.2 & 16.7 \\
Arbutus xalapensis & 54 & 72.0 & 19.4 & 10.8 \\
Quercus crassifolia & 35 & 46.7 & 12.5 & 7.4 \\
Pinus devoniana & 24 & 32.0 & 8.6 & 4.1 \\
Quercus castanea & 23 & 30.7 & 8.2 & 0.6 \\
Quercus affinis & 13 & 17.3 & 4.7 & - \\
Alnus acuminata & 2 & 2.7 & 0.7 & \\
Suma & 279 & 372.0 & 100.0 & 86.38 \\
\hline Total & 323 & 430.67 & - & - \\
\hline & & & &
\end{tabular}

En el Predio San Joaquín (Tabla 5) el estrato superior está compuesto por $P$. devoniana, $P$. teocote y $Q$. viminea con 20 árboles ha ${ }^{-1}$, lo que equivale al $5.4 \%$ de la zona, el estrato intermedio está compuesto por P. devoniana, P. teocote, P. pseudotrobus, $Q$. crassifolia, $Q$. obtusata y $Q$. resinosa con 138 árboles ha ${ }^{-1}$, lo cual corresponde al $37.5 \%$ de la zona, y el estrato inferior se conformó por $\mathrm{P}$. devoniana, A. xalapensis, P. teocote, P. pseudostrobus,
Q. crassifolia y $Q$. obtusata con 210 individuos ha ${ }^{-1}$, que equivale al $57 \%$ de la zona muestreada. Con respecto, al índice $\mathrm{A}$, tiene un valor de $\mathrm{A}=2.21$, una $A_{\max }=3.17$ y una $A_{r e l}=69.8 \%$ lo que indica que dentro del Predio San Joaquín tiene una distribución medianamente uniforme, ya que las especies con mayor valor de importancia se encuentran distribuidas dentro de los tres estratos, con altura promedio de 12.63 $\mathrm{m}$.

Tabla 5. Valores del índice vertical de Pretzsch para el Predio San Joaquín.

\begin{tabular}{|c|c|c|c|c|}
\hline \multicolumn{5}{|c|}{ Proporción (\%) } \\
\hline $\begin{array}{c}100 \%-80 \% \\
(29 m-23.2 m)\end{array}$ & $\mathrm{N}$ & $\mathrm{Nha}^{-1}$ & Del total & En la zona \\
\hline Pinus devoniana & 10 & 13.3 & 66.7 & 3.6 \\
\hline Pinus teocote & 4 & 5.3 & 26.7 & 1.4 \\
\hline Quercus viminea & 1 & 1.3 & 6.7 & 0.4 \\
\hline Suma & 15 & 20.0 & 100.0 & 5.4 \\
\hline \multicolumn{5}{|l|}{$\begin{array}{c}80 \%-50 \% \\
(23.2 \mathrm{~m}-14.5 \mathrm{~m})\end{array}$} \\
\hline Pinus devoniana & 53 & 70.7 & 51.0 & 19.1 \\
\hline Pinus teocote & 33 & 44.0 & 31.7 & 11.9 \\
\hline Pinus pseudostrobus & 11 & 14.7 & 10.6 & 4.0 \\
\hline Quercus crassifolia & 4 & 5.3 & 3.8 & 1.4 \\
\hline Quercus obtusata & 2 & 2.7 & 1.9 & 0.7 \\
\hline Quercus resinosa & 1 & 1.3 & 1.0 & 0.4 \\
\hline Suma & 104 & 138.7 & 100.0 & 37.5 \\
\hline \multicolumn{5}{|l|}{$\begin{array}{c}50 \%-0 \% \\
(14.5 m-0 m)\end{array}$} \\
\hline Pinus devoniana & 59 & 78.7 & 37.3 & 21.3 \\
\hline Arbutus xalapensis & 42 & 56.0 & 26.6 & 15.2 \\
\hline Pinus teocote & 25 & 33.3 & 15.8 & 9.0 \\
\hline Pinus pseudostrobus & 15 & 20.0 & 9.5 & 5.4 \\
\hline Quercus crassifolia & 10 & 13.3 & 6.3 & 3.6 \\
\hline Quercus obtusata & 7 & 9.3 & 4.4 & 2.5 \\
\hline Suma & 158 & 210.7 & 100.0 & 57.0 \\
\hline Total & 277 & 369.33 & - & - \\
\hline
\end{tabular}

\section{DISCUSIÓN}

Los bosques templados de San Luis Potosí, distribuidos dentro de la Sierra Madre Oriental, están dominados en el estrato arbóreo, principalmente por los géneros Quercus y Pinus. Para el estado, los estudios sobre la composición y diversidad de bosques templados son pocos y se han concentrado en ecosistemas prioritarios como Bosque Mesófilo de Montaña o Bosque de Niebla, para su conservación (LeijaLoredo et al. 2011, Fortanelli et al. 2014).

Al comparar la vegetación, se observa una similitud entre las familias presentes en de ambos 
sitios, para la UMA San Rafael que está dentro de Sierra de Álvarez se encontraron cuatro familias, mientras que para el Predio San Joaquín que se encuentra en la Sierra de Camarón se encontraron tres familias, siendo las más representativas en ambos sitios Pinacea y Fagacea, al respecto se sabe que la familia Pinacea es importante en el aprovechamiento forestal maderable (Flores et al. 2008). Se observa que la familia Fagacea es la más importante en la UMA San Rafael, lo que coincide con la menor cantidad de aprovechamiento maderable, ya que en la zona las especies más usadas para aprovechamiento maderable son de la familia Pinacea. Lo que muestra la diferencia entre el manejo forestal que se tiene en el predio San Joaquín, en el que la vegetación de mayor distribución es el bosque de pino-encino, con dominancia de la familia Pinacea, lo que indica que el predio San Joaquín tiene mayor desarrollo en el aprovechamiento maderable, por lo que se orienta a la regeneración o plantación de las especies de interés. Para la UMA San Rafael se registraron 10 especies y para el predio San Joaquín ocho especies, siendo el género Quercus el de mayor presencia, con seis y cuatro especies, seguido del género Pinus con dos y tres especies, respectivamente. Esto muestra que las áreas de estudio cuentan con similitud entre géneros y especies, ya que, de los cuatro géneros de la UMA San Rafael, tres también están en el predio San Joaquín (Quercus, Pinus y Arbutus). Al respecto López-Hernández (2017), reporta mayor presencia del género Pinus en bosques templados de Puebla, lo que coincide con Graciano-Ávila et al. (2017) para bosques templados del noreste de México.

Con respecto a las especies, $P$. teocote, $P$. devoniana, A. xalapensis, $Q$. crassifolia, $Q$. resinosa y $Q$. viminea, se encuentran en UMA San Rafael y el Predio San Joaquín. Esto muestra que existe un grado de similitud entre áreas, el efecto causado por la conservación de fauna dentro de la UMA San Rafael influye para que se realice una conservación de la vegetación arbórea que sirve como fuente de alimentación y refugio para la fauna, por lo cual se encuentran un mayor número de especies arbóreas (10 especies) que en el predio San Joaquín (ocho especies). La especie con mayor abundancia en el Pre- dio San Joaquín fue $P$. devoniana (44.86\%), aunque para la UMA San Rafael fue la tercera especie con mayor abundancia (14.04\%), esta especie tiene amplia distribución en la Sierra Madre Oriental, por lo que en México se utiliza para aserrío, para producción de pulpa de papel y resina, y es importante para fines de restauración, y en la economía local (Musálem y Sánchez-Cruz 2003).

Para la dominancia, el género con mayor porcentaje para ambos sitios fue Pinus con $48.89 \%$ para la UMA San Rafael y $82.95 \%$ para el Predio San Joaquín, este valor es mayor al reportado por Graciano-Ávila et al. (2017) dentro de bosques templados bajo manejo silvícola en el noreste de México. Dentro de la dominancia por especies destacan para ambos sitios $P$. teocote y $P$. devoniana con un área basal de 5.19 y $4.04 \mathrm{~m}^{2} \mathrm{~h}^{-1}$, respectivamente; para la UMA San Rafael y de 6.38 y 6.62 $\mathrm{m}^{2} \mathrm{~h}^{-1}$, correspondientemente para el Predio San Joaquín. Se observa una gran diferencia entre las dominancias en géneros y especie para las áreas de estudio, debido a que aunque dentro de la UMA San Rafael la vegetación se encuentra en estado de conservación, está se ve afectada en el crecimiento de diámetro por la competencia entre individuos del estrato arbóreo. En comparación, el predio San Joaquín cuenta con rodales bajo manejo silvícola, por lo cual cuentan con mayor espacio para el desarrollo de los individuos tanto en diámetro como en altura para así obtener un mayor volumen de producción.

El índice de valor de importancia (IVI), es un valor ponderado de la estructura de un bosque que se obtiene a partir del estudio de variables estructurales como la abundancia, la dominancia y la frecuencia. Este muestra que hay jerarquía entre las especies presentes en las áreas de estudio. Para la UMA San Rafael la especie dominante es $P$. teocote (IVI $=71.02)$ y las especies codominantes son $Q$. resinosa $(\mathrm{IVI}=53.85)$ y $P$. devoniana $(\mathrm{IVI}=42.93)$. Para el predio San Joaquín $P$. devoniana fue la dominante $(\mathrm{IVI}=98.92)$ y las codominantes son $P$. teocote (IVI $=76.01)$ y $P$. pseudostrobus $(\mathrm{IVI}=30.50)$ y de esta forma en ambas áreas se caracteriza un bosque pino-encino. Al respecto, en bosques similares a los estudiados Zacarías-Eslava (2011) determinaron 
el IVI para los bosque de encino-pino del Cerro del Águila en Michoacán, dentro del cual se distribuye $P$. devoniana (IVI = 23.36), mientras que Graciano-Ávila et al. (2017) menciona que $P$. teocote cuenta con un IVI de 19.41, en tanto que López - Hernández (2017) reportan para $P$. pseudostrobus un IVI de 3.58 , las especies arriba mencionadas coinciden en importancia con las encontradas dentro de las áreas de estudio, pero no con la especies dominantes, lo cual indica diferencia entre especies dominantes y codominantes a lo largo de la Sierra Madre Oriental.

El análisis de la diversidad de especies arbóreas muestra que la UMA San Rafael mostro la mejor diversidad en base al índice de Shannon con valor de 1.91, así como el mayor valor en el índice de Margalef con 1.55. Al compararlos con los obtenidos para el predio San Joaquín $\left(\mathrm{H}^{\prime}=1.51\right.$ y $\left.\mathrm{D}_{m g}=1.24\right)$ observamos que es evidente el efecto del manejo silvícola en esta última, aunque no es de carácter significativo este se puede explicar por la preferencia que se tiene por ciertas especies maderables dentro del Predio San Joaquín. Resultados similares fueron encontrados por Navar y González (2009), Hernández et al. (2013), Graciano-Ávila et al. (2017) y LópezHernández (2017) para bosques.

El índice $A$ tuvo valores similares para la UMA San Rafael y el Predio San Joaquín ( $A=2.19$ y 2.22 y $\mathrm{A}_{\max }=3.4$ y 3.2), lo que presenta uniformidad media en la diversidad de alturas. Los valores para $\mathrm{A}_{\text {rel }}$ son 64.3 y $69.8 \%$ respectivamente; valores de $A_{\text {rel }}$ cercanos al $100 \%$ indican que todas las especies se encuentran distribuidas de forma equitativa en los tres estratos de altura. Este resultado se explica, ya que para las dos áreas de estudio la mayoría de las especies se distribuyen dentro del estrato II y estrato III. Lo que coincide con lo reportado por Camacho et al. (2014) para bosques de pino-encino y GracianoÁvila et al. (2017) para bosques de Durango.

\section{CONCLUSIONES}

Dentro de la vegetación presente en las dos áreas de estudio destacan las familias Pinaceae y Fagaceae, con los géneros Pinus y Quercus. Las especies con mayor importancia son $P$. teocote, $P$. devoniana, P. pseudostrobus y $Q$. resinosa, destacando $P$. devoniana al ocupar mayor área basal, registrar el mayor número de árboles por hectárea y mayor frecuencia promedio. El bosque templado dentro de la UMA San Rafael presenta una mayor riqueza específica y diversidad arbórea que el distribuido en el predio San Joaquín, ya que este predio cuenta con un manejo silvícola de aprovechamiento maderable razón principal de la diferencia. Dentro de la estructura vertical el género Pinus es el más representativo, ya que se encuentra dentro de los tres estratos de altura para las dos áreas de estudio, la diversidad de altura se considera media y la etapa del bosque se observa como latizal, ya que la mayoría de los individuos presentan dimensiones medias por lo cual se ubican dentro de los estratos II y III.

\section{LITERATURA CITADA}

Aguirre COA (2002) Índices para la caracterización de la estructura del estrato arbóreo de ecosistemas forestales. Ciencia Forestal en México 27: 5-27.

Aguirre-Calderón OA (2015) Manejo forestal en el siglo XXI. Madera y Bosques 21: 17-28.

Camacho R, Alonso E, Gonzales-Tagle M A, Jimenez-Pérez J, Alanis-Rodriguez E, Ávila-Flores D Y (2014) Diversidad y distribución vertical de especies vegetales mediante el índice de Pretzsch. Ciencias UANL 17: 34-41

Castellanos-Bolaños JF, Treviño-Garza EJ, Aguirre-Calderón ÓA, Jiménez-Pérez J, Musalem-Santiago M, LópezAguillón R (2008) Estructura de bosques de Pino patula bajo manejo en Ixtlán de Juárez, Oaxaca, México. Madera y Bosques 14: 51-63.

Corral J, Aguirre OA, Jiménez J, Corral S (2005) Un análisis del efecto del aprovechamiento forestal sobre la 
diversidad estructural en el Bosque Mesófilo de Montaña "El Cielo", Tamaulipas, México. Investigaciones Agrarias: Sistema de Recursos Forestales 14: 217-228.

Flores RJD, Mireles SR, Flores CJA, Gonzáles SBM, Chapa VL (2008) Programa Estratégico Forestal del Estado de San Luis Potosí 2006 - 2025. Instituto Potosino de Investigación Científica y Tecnológica. San Luis Potosí, México. 204p.

Fortanelli MJ (1991) El suelo y los problemas de su uso en el estado de San Luis Potosí. Instituto de Investigaciones de Zonas Desérticas. Universidad Autónoma de San Luis Potosí. San Luis Potosí, México. 22p.

Fortanelli M J, García P J, Castillo L P (2014) Estructura y composición de la vegetación del Bosque de niebla de copalillos, San Luis Potosí, México. Acta Botanica Mexicana. Num 106. Pp. 161-186. Instituto de Ecología A.C. Patzcuaro, México.

Gadow KV, Zhang CY, Wehenkel C, Pommerening A, Corral-Rivas J, Korol M, et al. (2012) Forest structure and diversity. En: Pakkala T, von Gadow K (Eds.) Continuous cover forestry. Springer Netherlands. pp: 29-83.

García E (2004) Modificaciones al sistema de clasificación climática de Köppen. Instituto de Geografía. Universidad Autónoma de México. Quinta Edición, México D.F.

González MA, Schewendenm L, Jiménez J, Himmelsbach L (2007) Reconstrucción del historial de incendios y estructura forestal en bosques mixtos de pino Encino en la Sierra Madre Oriental. Madera y Bosques 13: 51-63.

González MA, Schwendemann L, Jiménez J, Schulz R (2008) Forest structure and woody plant species composition along a fire chronosequence in mixed pine-oak forest in the Sierra Madre Oriental, Northeast Mexico. Forest Ecology and Management 256: 161-167.

Graciano-Ávila G, Aguirre-Calderón OA, Alanís-Rodríguez E, Lujan-Soto JE (2017) Composición, estructura y diversidad de especies arbóreas en un bosque templado del Noreste de México. Ecosistemas y Recursos Agropecuarios 4: 535-542.

Hernández J, Aguirre O, Alanís E, Jiménez J, González MA (2013) Efecto del manejo forestal en la diversidad y composición arbórea de un bosque templado del noreste de México. Revista Chapingo serie Ciencias Forestales y del Ambiente 19: 189-199.

INEGI (2002) Síntesis de Información Geográfica del Estado de San Luis Potosí. 2da edición. Aguascalientes, México. 46p.

Jiménez J, Aguirre O, Kramer H (2001) Análisis de la estructura horizontal y vertical en un ecosistema multicohortal de pino-encino en el norte de México. Investigación Agraria: Sistema de Recursos Forestales 10: 355-366.

Leija-Loredo EG, Reyes-Hernández H, Fortanelli-Martínez J, Palacio-Aponte G (2011) Situación actual del bosque de niebla en el estado de San Luis Potosí, México. Investigación y Ciencia 19: 3-11.

Li Y, Hui G, Zhao Z, Hu Y, Ye S (2014) Spatial structural characteristics of three hardwood species in Korean pine broad-leaved forest-Validating the bivariate distribution of structural parameters from the point of tree population. Forest Ecology and Management 314: 17-25.

López-Hernández JA, Aguirre-Calderón OA, Alanís-Rodríguez E, Monarrez-Gonzalez JC, Gonzalez-Tagle MA, Jiménez-Pérez J (2017) Composición y diversidad de especies forestales en bosques templados de Puebla, México. Madera y Bosques 23: 1-16. 
Luna I, Morrone J J, Espinoza D (2004) Biodiversidad de la Sierra Madre Oriental. Comisión Nacional para el conocimiento y uso de la Biodiversidad. UNAM Primera Edición, México, D.F.

Magurran AE (2004) Measuring biological diversity. Blackwell. Cambridge, USA. 256p.

Margalef R (1958) Information theory in ecology. International Journal of General Sistems 3: 36-71.

Mora-Donjuán CA, Buendía-Rodríguez E, Rubio-Camacho EA, Alanís-Rodríguez E, Treviño-Garza EJ (2016) Distribución espacial, composición y estructura de un matorral en el Noreste de México. Revista Fitotecnia Mexicana 39: 87-95.

Mueller-Dombois D, Ellenberg H (1974) Aims and methods of vegetation ecology. Wiley, New York-Toronto. 547p.

Musálem MA, Sánchez-Cruz O (2003) Monografía de Pinus michoacana Martínez. Organización de las Naciones Unidad para la Alimentación y la Agricultura. Roma, Italia. http://agris.fao.org/agris-search/search.do?record ID=US201300098110. Fecha de consulta: 27 de noviembre de 2018.

Návar JJ, González GS (2009) Diversidad, estructura y productividad de bosques templados de Durango, México. Polibotánica 27: 71-87.

Ni R, Baiketuerhan YC, Zhang C, Zhao X, Gadow KV (2014) Analysing structural diversity in two temperate forests in northeastern China. Forest Ecology and Management 316: 139-147.

Pretzsch H (2009) Forest Dynamics, Growth and Yield. From Measurement to Model. Springer-Verlag Berlín Heidelberg. Alemania. 664p.

Rubio-Camacho EA, Gonzáñez-Tagle MA, Jiménez-Pérez J, Alanís-Rodriguez E, Avila-Flores DY (2014) Diversidad y distribución vertical de especies vegetales mediante el índice de Pretzsch. Revista Ciencias UANL 65: 34-41.

Shannon C (1948) The mathematical theory of communication. In: Shannon CE, Weaver W (Ed). The mathematical theory of communication. University of Illinois. Press Urbana. Illinois, USA. pp: 29-125.

Wehenkel C, Corral-Rivas JJ, Gadow KV (2014) Quantifying differences between ecosystems with particular reference to selection forests in Durango/Mexico. Forest Ecology and Management 316: 117-124.

Whittaker RH (1972) Evolution and measurement of species diversity. Taxon 21: 213-251.

Zacarias-Eslava L, Cornejo-Tenorio , Cortés-Flores J, González-Castañeda N, Ibarra-Manríquez G (2011) Composición, estructura y diversidad del cerro El Águila, Michoacán, México. Revista Mexicana de Biodiversidad 82: 854-869. 
